\title{
Healthy aging in elderly cochlear implant recipients: a multinational observational study
}

\author{
M. Marx ${ }^{1 *}$ D, I. Mosnier ${ }^{2}$, J. Belmin ${ }^{3}$, J. Wyss ${ }^{4}$, C. Coudert-Koall ${ }^{5}$, A. Ramos ${ }^{6}$, R. Manrique Huarte ${ }^{7}$, R. Khnifes ${ }^{8}$, \\ O. Hilly ${ }^{9}$, A. Martini ${ }^{10}$ and D. Cuda ${ }^{11}$
}

\begin{abstract}
Background: Given an increase in the aging population and its impact on healthcare systems, policy makers for provision of health and social services are aiming to keep older adults in good health for longer, in other words towards 'healthy aging'. Our study objective is to show that rehabilitation with cochlear implant treatment in the elderly with hearing impairment improves the overall health-related quality of life and general well-being that translate into healthy aging.
\end{abstract}

Methods: The multicentre, prospective, repeated measures, single-subject, clinical observational study will accrue 100 elderly, first-time, unilateral $\mathrm{Cl}$ recipients ( $\geq 60$ years) and analyze changes on specific measurement tools over ca. 20 months from preimplant to postimplant. Evaluations will consist of details collected through case history and interview questionnaires by clinicians, data logging, self-report questionnaires completed by the recipients and a series of commonly used audiometric measures and geriatric assessment tools. The primary indicator of changes in overall quality of life will be the HUI-3.

Discussion: The protocol is designed to make use of measurement tools that have already been applied to the hearing-impaired population in order to compare effects of $\mathrm{Cl}$ rehabilitation in adults immediately before their implantation, (pre-implant) and after gaining 1-1.5 years of experience (post-implant). The broad approach will lead to a greater understanding of how useful hearing impacts the quality of life in elderly individuals, and thus improves potentials for healthy aging. Outcomes will be described and analyzed in detail.

Trial registration: This research has been registered in ClinicalTrials.gov (http://www.clinicaltrials.gov/), 7 March 2017 under the $n^{\circ}$ NCT03072862.

Keywords: Cochlear implant, Data logging, Hearing-related healthy aging, Hearing loss in elderly

\footnotetext{
* Correspondence: marx.m@chu-toulouse.fr

'Otology and Neurotology Department, ENT Department, Bâtiment Pierre

Paul Riquet - Hôpital Purpan, Place du Dr Baylac, 31059 Toulouse Cedex 9,

France

Full list of author information is available at the end of the article
}

(c) The Author(s). 2020 Open Access This article is licensed under a Creative Commons Attribution 4.0 International License, which permits use, sharing, adaptation, distribution and reproduction in any medium or format, as long as you give appropriate credit to the original author(s) and the source, provide a link to the Creative Commons licence, and indicate if changes were made. The images or other third party material in this article are included in the article's Creative Commons licence, unless indicated otherwise in a credit line to the material. If material is not included in the article's Creative Commons licence and your intended use is not permitted by statutory regulation or exceeds the permitted use, you will need to obtain permission directly from the copyright holder. To view a copy of this licence, visit http://creativecommons.org/licenses/by/4.0/ The Creative Commons Public Domain Dedication waiver (http://creativecommons.org/publicdomain/zero/1.0/) applies to the data made available in this article, unless otherwise stated in a credit line to the data. 


\section{Background}

Worldwide demographic shifts indicate that the burden of an aging population on healthcare systems will be affected by the number of babies being born into the more industrialized populations. That can be illustrated by census findings that reveal both Europe and Asia have the largest number of individuals over the age of 65 but the lowest birthrates. The US is also approaching a similar demographic. This means that the contribution to tax and social systems that fund healthcare supplied by working-age individuals will not be able to contribute enough to help finance the healthcare needs of the elderly because those contributing will be too few [58]. The PRB further reports that by 2050, individuals older than 80 years of age could reach as many as $20 \%$ of the European population. The challenge, then, is to provide means and methods that improve the health conditions of the elderly enabling them to be gainfully active as healthy-aging individuals. Utilization of effective hearing capacity may contribute to an individuals' general health status.

In terms of healthy aging, defined by WHO (World Health Organization) as "The process of developing and maintaining functional ability that enables wellbeing in older age" [46], simply living longer is not a successful endpoint; it must include the quality of life (QoL) during a person's extended longevity [6]. Dalton et al. [17] were one of the earliest researchers to point out specifically that hearing loss greatly affects quality of life in the elderly. We now understand that it may have a cascading effect [8] and interact negatively $[6,23,55]$ with physical, cognitive and psychosocial conditions.

The presence of a HL and its considerable effects on quality of life and healthy aging has been established in various epidemiological studies [18, 27, 43], reviews [4, $10,11,69]$, meta-analyses [38, 45, 68]; and a large number of clinical studies. Contrera et al. [13] in their prospective study that enlisted both HA and CI users over the age of 50 years, concluded that CIs were more effective than HA with respect to QoL outcomes, also recognized by others as summarized in the review provided by Schaefer et al. [64]. Importantly, hearing loss and its associated effects are considered modifiable with today's technologies [19, 28, 32, 52, 53]. Lin [43] provides a comprehensive overview about the pervasive influence of hearing loss on cognition and other domains.

Laureyns M. [37] estimated that in Europe alone, by 2025, 114 million citizens will be over the age of 65 years with 61 million self-reporting $\mathrm{HL}$; that is, $54 \%$ of that older age group. Such an incidence significantly impacts the socioeconomic burden on societies. These data include only those who report hearing loss, but the number could be significantly larger as its insidious nature as a hidden disability usually develops slowly and may not necessarily be recognized. Individuals may choose to ignore $\mathrm{HL}$ as it may represent a stigma associated with getting old. Additionally, HL is considered a normal part of aging and, therefore, not worth reporting. These reasons highlight the importance, and urgency, to uncover and confirm the most promising methods and techniques that support healthy aging, especially by addressing currently available treatments that may modify debilitating effects such as HL.

The most common path to remediating hearing loss is through evaluations that may lead to the use of hearing aids (HA). However, in cases where HL cannot be compensated by hearing aids, cochlear implant $(\mathrm{CI})$ may be a viable option [63]; however, due to financial constraints, CIs may not always be available. Thus, already in 2017, in a report called Action for Hearing Loss, WHO urged international investment in the identification and treatment of HL enabling improved access to cochlear implants, among other auditory enhancement technologies [74]. There appears to be no age limit to receiving a CI $[66,75]$. The oldest recipient, to our knowledge, to receive a Nucleus/Cochlear CI was 102 years old.

With respect to benefits from cochlear implantation, strong supporting evidence suggests that an improvement in functional hearing status via the use of cochlear implants in older adults has far-reaching effects on hearing-related quality of life and other health-related domains. Earlier studies (2002-2009) were most interested in psychosocial factors such as depression and isolation [22, 51, 57, 72]; whereas, most of the later studies (2015-2019) began investigating effects on cognition [5, 9, 12, 14, 47-49, 62, 63, 66, 73]. Routine clinical follow up shows a trend for added benefits of CI treatment upon dependency, mobility and the risk of falls [35]. It should be noted that all the aforementioned studies that performed pre-post implant analyses concluded that $\mathrm{CI}$ benefits were observed in all areas evaluated for the study, although elderly user's outcomes scores may have been lower than those of comparable normal-hearing adults [12]. Outcomes from longitudinal studies conducted in an aging population suggest that CI implantation may help to maintain cognitive function and delay its decline [31, 42, 48].

Benefits associated with quality of life as a primary aim of investigation have been studied [22]. was the only study to report solely on QoL, while other researchers considered additional areas that contribute to QoL [7, 73]. The current study, to our knowledge, is the only international prospective investigation currently researching the primary impact on quality of life of cochlear implantation. A similar study underway in Australia [63], aiming for 5year follow up, has the primary goal of examining cognitive change associated with CI rehabilitation and utilizes a very different test battery with only two evaluation tools in 
common. The first report on outcomes confirms benefit in all areas for an initial cohort of $20 \mathrm{CI}$ users with 18 month's listening experience.

Here, we describe the study design of a multicenter prospective study in a large cohort of elderly individuals with equivalent $\mathrm{CI}$ experience. As to its unique features, it utilizes a full array of tests that include assessments in the physical, mental, psychosocial domains but with the primary focus on QoL.

\section{Methods and design}

\section{Study aims}

The primary aim is to identify significant improvement in the overall health-related quality of their life compared to their preimplant condition as measured by the HUI-3. The secondary aim is to investigate the influence of cochlear implant use on a variety of healthy-aging domains and, therefore, the overall well-being of elderly individuals. Specific healthy-aging domains to be investigated are hearing ability and communication, dependency, cognition, risk of falls, loneliness and depression/mood. Further, the study considers the impact of consistent daily use of CI communication through evaluation of data-logging outcomes.

\section{Study design}

A repeated measure, single-subject design assesses the changes in health-related quality of life and overall wellbeing as the primary end-point of the study. As an observational study, no additional intervention is applied to our specific population of CI recipients. The study design is multicentre and includes language-appropriate materials relative to the participating $\mathrm{CI}$ recipient and has been registered at Clinicaltrials.gov identifier: NCT03072862.

\section{Timing schedule}

The evaluation protocol is designed to coincide with routine clinical visits. Each subject will be assessed with the full battery of tests that are repeated at each of the three visits (Pre1, Post1, Post2, see Fig. 1). The maximum administration time for completion of all measures is estimated to be approximately two hours of which one hour is dedicated to standard self-report forms completed by the CI recipient.

\section{Subjects}

Study participants who have agreed to a first-time unilateral implant will receive an appropriately-chosen commercially available Nucleus ${ }^{\oplus}$ Cochlear Implant Systems (Cochlear Ltd., Sydney, Australia) with the most recent implant technologies that are compatible with processors offering data logging. Subjects are aged $\geq 60$ years with hearing loss that meets all local criteria for CI

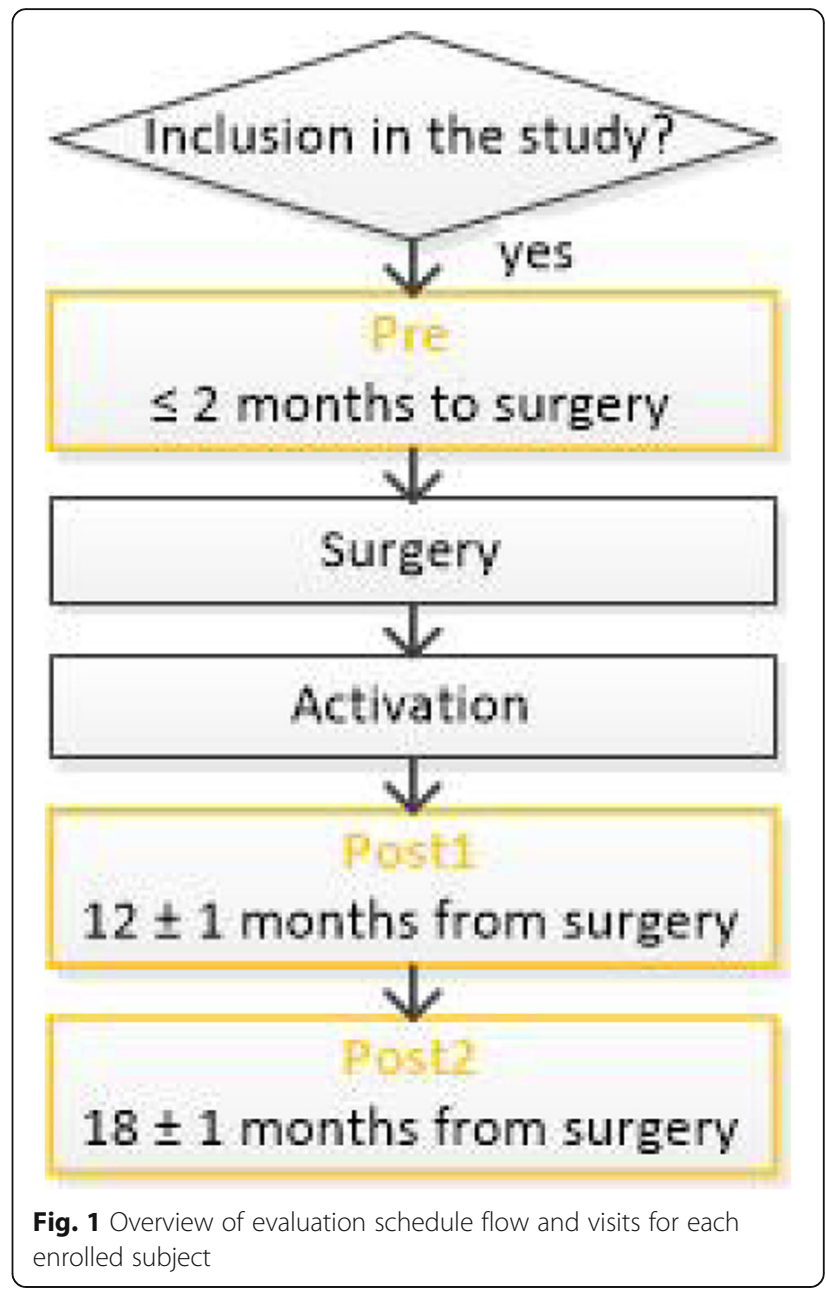

treatment. Subjects are enrolled into the clinical investigation only after signing the Patient Informed Consent Form prior to the pre-implant assessment (baseline, V1). The expected duration for each subject from enrolment is 20 months (+/-1 month). The study aims to accrue participants distributed across all study sites and languages to avoid site and cultural bias. A maximum of 100 individuals are to be included.

Selection Criteria:

Inclusion:

- Unilateral CI candidates with bilateral postlingual deafness with intention to treat

- Contralateral ear: average pure tone thresholds indicate a moderately-severe to profound hearing loss (4 freq. Average: $0.5,1,2$ and 3 or $4 \mathrm{kHz}>56$ dBHL).

- Willingness to participate in and to comply with all study procedures

- Fluency in languages used to assess clinical performance

- Appropriate expectations from routine CI treatment 
- Able to decide on study participation personally, and independently sign their consent

Exclusion:

- Significantly/severely dependent or fragile

- Unable to provide consent personally

- Unable to complete questionnaires for selfassessment independently

- Unilateral hearing loss

- Recipients of sequential and simultaneous bilateral CI

- Ossification or other cochlear anomalies preventing full electrode insertion or medical contraindications to surgery

- Retro cochlear or central origins of hearing impairment

- Significant comorbidities preventing study participation (e.g. blindness, immobility or in a wheel chair, severe aphasia, and other)

- Clinic Standard fail criteria for CI candidacy in regards to chronic depression, dementia, and cognitive disorders

- Unrealistic expectations on the part of the subject regarding the possible benefits, risks and limitations inherent to the procedure and prosthetic device.

\section{Materials}

Demographic and hearing impairment characteristics, such as the aetiology of deafness, the duration of profound hearing loss, the hearing aid use, will be collected. The education level will be assessed using the ISCED (International Standard Classification of Education). In addition to these, general healthcare and patient-profile data and a selection of observational clinical assessment tools were chosen for repeated evaluations of the overall health status of the elderly individual at pre- and post-CI treatment intervals. The measures are commonly used in audiology and/or geriatric practices and are administered in the appropriate language. Translations of questionnaires have been controlled for via a validated translation process thus enabling collation of the data gathered cross culturally.

A short description of assessment tools administered at baseline are shown in Table 1 and listed alphabetically, below. The whole evaluation is performed in the subject's individual daily listening condition, typically with unilateral or bilateral optimally fitted hearing aids before cochlear implantation, and with the cochlear implant plus a contralateral hearing aid when residual hearing in the non-implanted ear can be optimized, after the procedure.

\section{Audiometric assessments (routine)}

These include standard unaided ( $\mathrm{dB}$ HL) and aided ( $\mathrm{dB}$ SPL) threshold measures for frequencies $250-4000 \mathrm{~Hz}$ and speech discrimination in quiet and noise.

Categories of Auditory Perception II (CAP-II) is an auditory skill rating index consisting of nine hierarchical categories completed by the clinician as an observation of the individuals hearing abilities. The auditory skills increase in complexity ranging from perception of environmental sounds to telephone conversation with an unfamiliar speaker [25].

Data Logging: Cochlear sound processors feature an in-built data-logging function. Specifics of the data to be collected, along with anonymous identifying reference, include the average time on-air per day and average times in noise, speech in noise, speech, quiet, music and wind; i.e. product usage data including the use of product features and acoustic environments [60].

Table 1 Healthy-aging domains and assessment tools used for evaluation

\begin{tabular}{|c|c|c|c|c|c|c|c|c|c|c|c|c|c|c|c|c|}
\hline \multirow[t]{2}{*}{ Domain } & \multicolumn{3}{|c|}{ Clinician report } & \multicolumn{4}{|c|}{ Self-report by recipient } & \multicolumn{4}{|c|}{ Routine Audiology } & \multicolumn{4}{|c|}{ Routine Geriatric } & \multirow{2}{*}{$\begin{array}{l}\text { Device } \\
\text { Usage } \\
\text { Data } \\
\text { Logging }\end{array}$} \\
\hline & $\begin{array}{l}\text { CAP- } \\
\|\end{array}$ & $\begin{array}{l}\mathrm{L}- \\
\mathrm{IADL}\end{array}$ & DeJong & $\begin{array}{l}\mathrm{HUI-} \\
3\end{array}$ & $\begin{array}{l}\text { GDS- } \\
15\end{array}$ & $\begin{array}{l}\mathrm{HHIE-} \\
\mathrm{S}\end{array}$ & SSQ & PTA & SFT & $\begin{array}{l}\text { Sp- } \\
\text { Q }\end{array}$ & $\begin{array}{l}\text { Sp- } \\
N\end{array}$ & DDST & MMSE & $\begin{array}{l}\text { Trail } \\
\text { B }\end{array}$ & TUG & \\
\hline Hearing & $\bullet$ & & & & & $\bullet$ & & $\bullet$ & $\bullet$ & $\bullet$ & 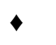 & & & & & \\
\hline Communication & $\bullet$ & & & $\bullet$ & & $\bullet$ & $\bullet$ & & & & & & & & & situations \\
\hline Risk of Falls & & & & & & & & & & & & & & & $\bullet$ & \\
\hline Cognition & & & & $\bullet$ & & & & & & & & $\bullet$ & $\bullet$ & $\bullet$ & & \\
\hline $\begin{array}{l}\text { Loneliness /Social } \\
\text { Isolation }\end{array}$ & & & $\bullet$ & & & & $\bullet$ & & & & & & & & & \\
\hline Dependency & & $\bullet$ & & & & & & & & & & & & & & \\
\hline Depression/Mood & & & $\bullet$ & $\bullet$ & 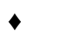 & & & & & & & & & & & \\
\hline General Health & & & & $\bullet$ & & & & & & & & & & & & \\
\hline Device Use & & & & & & & & & & & & & & & & time \\
\hline
\end{tabular}


De Jong Loneliness is the six-item validated version [20] with three questions assessing social isolation and three on emotional loneliness. The scale is completed via interview.

Digit-Symbol Substitution Test (DSST) is pencil and paper test in which the subject is given a key grid of numbers and matching symbols (e.g. $1 /-, 2 / \perp \ldots 7 / \Lambda$, $8 / \mathrm{X}, 9 /=)$ and a test section with numbers and empty boxes. The task is to fill-in as many empty boxes as possible with a symbol matching each number. The score is the number of correct number-symbol matches achieved in $120 \mathrm{~s}$.

Geriatric Depression Scale-15 (GDS-15) is a selfadministered questionnaire to record symptoms of depression in older adults. For each of the 15 questions of the scale, the subjects answer Yes or No, where ten questions indicate the presence of depression when answered positively while the other five are indicative of depression when answered negatively [65].

Hearing Handicap Inventory in the Elderly Screening test (HHIE-S) is a short form, selfassessment scale designed to indicate the effects of hearing impairment on emotional and social adjustment in everyday life of the elderly individual [50].

Health Utility Mark III (HUI3) is applied to assess health-related quality of life and is completed by the recipient. It considers, in this case, the impact of CI treatment across eight health domains (vision, hearing, speech, ambulation, dexterity, emotion, cognition and pain) [21].

Lawton Instrumental Activities of Daily Living Scale (L-IADL) is completed by the clinician mainly to determine a person's ability to care for him or herself without any help from others. It measures the functional impact of emotional, cognitive, and physical impairments and their need for personal care services [39].

Mini Mental State Examination (MMSE)-Short formwas derived from the six memory domains of the full MMSE. It can be used to estimate the severity and progression of cognitive impairment and cognitive change in an individual over time [29].

Speech Spatial Qualities (SSQ) is a self-assessment scale of hearing ability and communication in daily environments, completed by the patient. It is divided into the three subcategories of speech (comprehension), spatial (hearing in space) and quality (speech and sounds) [24].

The Timed Up and Go test (TUG) measures the time a person takes to stand up from a standard armchair, walk three meters, turn around, walk back to the chair and then sit down again [56].

The Trail Test B (TMT-B) is neuro physiological test assessing executive function. It is a more cognitively demanding task than the Trail A test [70].

\section{Monitoring the study}

The study is monitored by an external clinical research organization. A clear data management program has been set into place to validate and verify electronic data capture. Study sites and documentation may be subject to Quality Assurance audits during the course of the observational study. In addition, regulatory bodies, at their discretion, may conduct inspections, during and after study completion.

\section{Statistical considerations}

For primary and secondary study objectives, an intra subject endpoint comparison is used: All pairwise comparisons are of interest; i.e. preimplant (Pre)-to-12 months postimplant (Post1), Pre-to-18 months postimplant (Post2), and the change from Post 1 to Post 2.

\section{Sample size calculation}

The HUI3 will serve as the primary outcome measure for this study to determine the added health utility gain for the multi- attribute health status between Pre, Post1 and Post2. A one-way ANOVA will be performed on HUI3 multi-attribute scores, with visit as the main factor. Post-hoc tests will be performed to determine significant differences between visits.

In [71] the authors suggest that a gain of 0.1 in HUI3 multi attribute score is considered highly clinically significant. In addition, the UKCISG paper gives data on the variation in HUI3 gain across age groups receiving cochlear implants: the $95 \%$ confidence intervals for gain in HUI3 allow us to estimate the standard deviation of change of subjects aged $60+$ as 0.25 . Assuming a paired t-test of the difference in HUI3 multi attribute score, then with $90 \%$ power and a $5 \%$ level of significance, a mean difference of 0.1 would require 68 participants based on a standard deviation of change equal to 0.25 . Because all three pairwise tests between visits are of interest Bonferroni corrections will be applied. The recomputed sample size required with this significance level requires at least 88 participants to find a change of 0.1 units in the HUI3 significance. Taking attrition into account, the inclusion of 100 study participants was deemed appropriate.

\section{Discussion}

Our study objective is to show the potential for positive effects of $\mathrm{CI}$ treatment according to local indication criteria on overall health-related quality of life and general well-being that ultimately translate into healthy aging. The study outcomes are intended to provide transparent and comparable, evidence-based information to healthcare policy makers in support of guiding informed decisions on the provision of health services for the treatment of hearing loss. 
In 2014, Prince et al. [59] published a report in Lancet on the leading contributors to the burden of disease in people aged $>60$ years; in particular, mental and neurological disorders. Burden of disease in the aging population has a high economic impact for society. Therefore, we may assume that through CI treatment for permanent deafness, there is a potential to decrease the burden of disease in aging adults by improving important social, health and cognitive functions in addition to restoring hearing function.

The comprehensive protocol considers a range of widely accepted, interrelated metrics associated with aging beyond functional hearing. Use of this protocol does not propose to investigate causal effects but rather the pre-post implant status of $\mathrm{CI}$ candidates who have 1-1.5 years of experience using their unilateral implants. In line with existing study designs for $\mathrm{CI}$ recipients, we have chosen both self-report and clinician-report questionnaires that are essentially non-verbal, thus removing challenges directly associated with utilizing newly acquired functional hearing [32]. All the standardized tests have been applied to $\mathrm{CI}$ recipients in published research with the exception of the Lawton iADL and the DSST both of which have been used in assessing hearingimpaired individuals with hearing aids [3, 43].

The HUI-3, as the primary instrument in assessing CI implant treatment effects on overall health-related quality of life of elderly individuals, has been recommended as the most sensitive and useful tool for CI users $[1,16,34,61]$. General functional hearing is addressed by audiometric evaluations and the CAP-II, which is most often used in a younger hearing-impaired population. However, it has also been applied to adult CI users [54, 72].

Further, the protocol investigates the major categories of psychosocial, mental, physical and communication behaviors with a range of other assessment tools currently applied to hearing-impaired individuals. The psychosocial domain regarding the issue of depression has been measured by the GDS-15 in CI users [9, 57]. Dependency and loneliness are assessed by the deJong Loneliness Scale that has yielded results in studies of CI users [7] and the Lawton in HA users [3]. Cognition is evaluated with the MMSE in CI studies $[36,47,48,62]$ and the Trail B by $[49,73]$ in CI. The Digit-Symbol has been applied to HA users [43] but it has also been recommended for CI users [30]. The physical domain focuses on the tendency to fall and is addressed by the TUG applied to CI users [2, 40]. Finally, general communication is addressed by the SSQ previously used in CI users [41, 51, 76], and the HHIE. The HHIE was included as it is one of the most widely applied questionnaires with respect to auditory participation [67] and has been reported with CI users [44].
Data logging is an important addition as it may provide new insights and potential correlations with healthy-aging domains. As has been pointed out by [4], there have been no studies, to our knowledge, investigating the impact of $\mathrm{CI}$ and their relationship to complex social environments. Data logging may serve as an initial step by enabling comparison of different sound environments (scenes), especially quiet versus noise [15]. A further advantage of data logging is use time. Several authors have posited that hours of auditory input or CI use per day have a significant effect on socialization and other psychosocial outcomes [26, 33]. Although data logging first became available in 2013, no studies have correlated outcomes with impact on quality of life.

A potential limitation of the study is the lack of a control group. In fact, establishing a randomized-controlled study design is not straightforward as it would imply the need not to give a CI to those control patients who meet criteria for cochlear implantation, which is far from ethical. The single-subject, repeated-measures design allows for subjects to serve as their own controls, and thus increases statistical power. But, the lack of preoperative repeated measures does not allow an estimate of withinsubject variations in a non-intervention state. To counter this, the relatively large population size allows for a good estimate of effect size and would make the results broadly applicable. Another limitation is that the investigator or their team may have access to preoperative evaluation information at the time of the follow-up evaluations. However, many of the test results require coding in order to obtain a score and places a layer of protection against investigators making comparisons at the time of evaluation.

Ultimately, the study aim is to provide clinical evidence that $\mathrm{CI}$ treatment in aging adults currently has the potential to improve the overall health status including, but not exclusive to, hearing function, which in turn can create cost savings from a payer and societal perspective. Age-related HL is often ignored as a stigma associated with aging or just accepted as a natural side-effect of it. Therefore, growing evidence may remove these boundaries and support referring professionals and potential CI candidates in their decision for $\mathrm{CI}$ treatment as soon as significant hearing loss is diagnosed in the hopes of reducing the overall impact of hearing disability.

\section{Abbreviations}

CAP-II: Categories of Auditory Perception II; Cl: Cochlear implant; DSSTDigit: Symbol Substitution Test; GDS-15: Geriatric Depression Scale-15; HA: Hearing aids; HHIE-S: Hearing Handicap Inventory in the Elderly Screening test; L-IADL: Lawton Instrumental Activities of Daily Living Scale; MMSE: Mini Mental State Examination; PRB: Population Reference Bureau; PTA: Pure tone Audiometry; QoL: Quality of life; SFT: Sound Field Thresholds; SSQ: Speech Spatial Qualities; TMT-B: Trail Test B; TUG: The Timed Up and Go test; WHO: World Health Organization 


\section{Acknowledgements}

We would like, in advance, to thank all participating implant recipients who will take the time to undergo evaluations throughout the study and to thank the clinicians in all participating clinics involved in providing data to be used in the analysis of study outcomes. As external consultant, Prof. Michal Luntz contributed to the design of the study protocol.

\section{Trial status}

The trial is currently in recruitment phase.

\section{Authors' contributions}

All authors (MM, IM, JB, JW, CCK, ARM, RMH, RK, OH, AM, DC) made a substantial contribution to the conception and the design of the study. Author JW drafted the study protocol and author CCK managed the clinical project. All authors (MM, IM, JB, JW, CCK, ARM, RMH, RK, OH, AM, DC) revised and approved the final version of the manuscript.

\section{Funding}

This study is sponsored by the company Cochlear. Sponsorship covers development of the protocol, licensing and translation and development of study documents, monitoring, insurance, ethic committee fees and publications. The study was designed by the sponsor in collaboration with an independent biostatistician. Data are collected via the sponsor's electronic data capture system, will be analyzed by an independent statistician, and the results will be interpreted by the sponsor's own experts in collaboration with the principal investigators. Manuscripts will be drafted by consultant medical writers and the sponsor's own experts in collaboration with the principal investigators. Clinics are reimbursed for their efforts in conducting the study protocol.

\section{Availability of data and materials}

Data generated from the current study will be made available to study investigators on reasonable request. Study investigators may publish their local data separately. Overall outcomes of the study are intended for publication in peer-reviewed journals and proceedings, professional forums, international presentations and lectures and other dedicated communications. As well as grouped findings, each participating clinic may choose to disseminate their own data via local media.

\section{Ethics approval and consent to participate}

Each collaborating clinic has obtained Ethics Committee (EC) Approval/ Opinion (or Competent Authority (CA) Approval if applicable) for their participation in the study and has obtained formal local approval prior to enrolling their first subject: comité de Tours - Centre-ouest1 for Paris and Toulouse (2017 T2-17 (2017-A01964-49); Ethics committee Bnai Zion Medical center for Haïfa (0074-17-BNZ); Ethics committee Rabin MC Belinson Hospital for Petah Tikva; Comitato Etico A.USL Di Piacenza (5987/2017); Comitato Etico Clinica della Provincia Di Padova (4295/AO/17); Comité de Etica de la Investigacion del complejo Hospitalario Insular-Materno Infantil de Las Palmas; Comité Etico de Investigacion Clinica de Navarra for Pamplona (EO17/1). Subjects are enrolled into the clinical investigation only after signing the Patient Informed Consent Form prior to the pre-implant assessment. The study is conducted in accordance with the most recent version of the Declaration of Helsinki, the EN ISO 14155:2011 and any regional or national regulations, as appropriate.

\section{Consent for publication}

No private information of patients is intended for publication.

\section{Competing interests}

This study is sponsored by the company Cochlear. The results will be interpreted by Cochlear's own experts in collaboration with the principal investigators.

\section{Author details}

'Otology and Neurotology Department, ENT Department, Bâtiment Pierre Paul Riquet - Hôpital Purpan, Place du Dr Baylac, 31059 Toulouse Cedex 9, France. ${ }^{2}$ Groupe Hospitalier de la Pitié-Salpétrière, Paris, France. ${ }^{3}$ Université Pierre and Marie Curie and Hôpital Charles Foix, Paris, France. ${ }^{4}$ Cochlear Ltd., Sydney, Australia. ${ }^{5}$ Cochlear AG, Basel, Switzerland. ${ }^{6}$ Complejo Hospitalario Universitario Insular Materno Infantil, Las Palmas de Gran Canaria, Spain.
${ }^{7}$ Clinica Universitaria de Navarra, Pamplona, Spain. ${ }^{8}$ Bnai Zion Medical Center, Haifa, Israel. ${ }^{9}$ ENT Department at Rabin Medical Center (Beilinson), Petah Tikva, Israel. ${ }^{10}$ ENT Otosurgery Department at Azienda Ospedaliera di Padova, Padova, Italy. ${ }^{11}$ ENT Department of Ospedale Guglielmo da Saliceto, Piacenza, Italy.

Received: 4 February 2020 Accepted: 24 June 2020

Published online: 23 July 2020

\section{References}

1. Arnoldner C, Lin VY, Bresler R, Kaider A, Kuthubutheen J, Shipp D, Chen JM. Quality of life in cochlear implantees: comparing utility values obtained through the medical outcome study short-form survey-6D and the health utility index mark 3. Laryngoscope. 2014;124(11):2586-90.

2. Baudhuin, JL. Balance function following cochlear impelantation PhD dissertation. Wash Uni School of Med, Program in Audiology and Communication Sciences; 2008. [http://digitalcommons.wustl.edu/pacs capstones/173]. Accessed 25 Jul 2019

3. Boi R, Racca L, Cavallero A, Carpaneto V, Racca M, Dall' Acqua F, et al. Hearing loss and depressive symptoms in elderly patients. Geriatr Gerontol Int. 2012;12(3):440-5.

4. Borik, Y. Social isolation, social and emotional loneliness, and self-reported hearing difficulties in older adults with hearing loss. PhD Dissertation 2016. [https://academicworks.cuny.edu/gc_etds/1330]. Accessed 26 Jul 2019.

5. Bosdriesz JR, Stam M, Smits C, Kramer SE. Psychosocial health of cochlear implant users compared to that of adults with and without hearing aids: results of a nationwide cohort study. Clin Otolaryngol. 2018;43(3):828-34.

6. Browning C, Thomas S, Kendig H, Ory MG. Healthy Ageing. In: Pachana NA, editor. Encyclopedia of Geropsychology. Springer Science + Business Media; 2016. p. 1-9.

7. Brüggemann P, Szczepek AJ, Klee K, Gräbel S, Mazurek B, Olze H. In patients undergoing Cochlear implantation, psychological burden affects tinnitus and the overall outcome of auditory rehabilitation. Front Hum Neurosci. 2017:5(11):226.

8. Campos J, Ramkhalawansingh R, Pichora-Fuller MK. Hearing, self-motion perception, mobility, and aging. Hear Res. 2018;369:42-55.

9. Castiglione A, Benatti A, Velardita C, Favaro D, Padoan E, Severi D, et al. Aging, Cognitive Decline and Hearing Loss. Effects of Auditory Rehabilitation and Training with Hearing Aids and Cochlear Implants on Cognitive Function and Depression among Older Adults. Audiol Neurootol. 2016;21(Suppl 1):21-8.

10. Cherko M, Hickson L, Bhutta M. Auditory deprivation and health in the elderly. Maturitas. 2016;88:52-7.

11. Davis A, McMahon CM, Pichora-Fuller KM, Russ S, Lin F, Olusanya BO, et al. Aging and Hearing health: The Life-course Approach. Gerontologist. 2016; 56(Suppl 2):S256-67.

12. Claes AJ, Van de Heyning P, Gilles A, Hofkens-Van den Brandt A, Van Rompaey V, Mertens G. Impaired Cognitive Functioning in Cochlear Implant Recipients Over the Age of 55 Years: A Cross-Sectional Study Using the Repeatable Battery for the Assessment of Neuropsychological Status for Hearing-Impaired Individuals (RBANS-H). Front Neurosci. 2018;12:580.

13. Contrera KJ, Betz J, Li L, Blake CR, Sung YK, Choi JS, Lin FR. Quality of life after intervention with a cochlear implant or hearing aid. Laryngoscope. 2016;126(9):2110-5

14. Cosetti MK, Pinkston JB, Flores JM, Friedmann DR, Jones CB, Roland JT Jr, Waltzman SB. Neurocognitive testing and cochlear implantation: insights into performance in older adults. Clin Interv Aging. 2016;11:603-13.

15. Cristofari E, Cuda D, Martini A, Forli F, Zanetti D, Di Lisi D, et al. Multicenter clinical evaluation of data logging in Cochlear implant recipients using automated scene classification technologies. Audiol Neurootol. 2017;22(4-5): 226-35.

16. Crowson MG, Semenov YR, Tucci DL, Niparko JK. Quality of life and costeffectiveness of cochlear implants: A narrative review. Audiol Neurotol. 2017; 22:236-58 [Review].

17. Dalton DS, Cruickshanks KJ, Klein BE, Klein R, Wiley TL, Nondahl DM. The impact of hearing loss on quality of life in older adults. Gerontologist. 2003; 43(5):661-8.

18. Dammeyer J, Chapman M. Prevalence and characteristics of self-reported physical and mental disorders among adults with hearing loss in Denmark: a national survey. Soc Psychiatry Psychiatr Epidemiol. 2017;52(7):807-13. 
19. Dawes P. Hearing interventions to prevent dementia. HNO. 2019;67(3):165-71.

20. de Jong GJ, van Tilburg TG. A 6-item scale for overall, emotional, and social Ioneliness: confirmatory tests on survey data. Res Aging. 2006;28:582-98.

21. Feeny D, Furlong W, Torrance GW, Goldsmith CH, Zhu Z, DePauw S, et al. Multi-attribute and single-attribute utility functions for the health utilities index mark 3 system. Med Care. 2002;40(2):113-28.

22. Francis HW, Chee N, Yeagle J, Cheng A, Niparko JK. Impact of cochlear implants on the functional health status of older adults. Laryngoscope. 2002;112(8 Pt 1):1482-8.

23. Francis HW, Yeagle JA, Thompson CB. Clinical and psychosocial risk factors of hearing outcome in older adults with cochlear implants. Laryngoscope. 2015;125(3):695-702

24. Gatehouse S, Noble W. The speech, spatial and qualities of hearing scale (SSQ). International journal of audiology. Int J Audiol. 2004;43(2):85-99.

25. Gilmore, L. The Inter-Rater Reliability of Categories of Auditory PerformanceII (CAP)-II. PhD thesis. University of Southampton, Faculty of Engineering, Science, Mathematics, Institute of sound and vibration research. 2010. [https://eprints.soton.ac.uk/173775/]. Accessed 12 Jun 2019.

26. Giroud N, Lemke U, Reich P, Matthes KL, Meyer M. The impact of hearing aids and age-related hearing loss on auditory plasticity across three months - an electrical neuroimaging study. Hear Res. 2017;353:162-75.

27. Goman AM, Lin FR. Hearing loss in older adults - from epidemiological insights to national initiatives. Hear Res. 2018;369:29-32.

28. Gopinath B, Wang JJ, Schneider J, Burlutsky G, Snowdon J, McMahon CM, et al. Depressive symptoms in older adults with hearing impairments: the Blue Mountains study. J Am Geriatr Soc. 2009;57(7):1306-8.

29. Haubois G, Annweiler C, Launay C, Fantino B, de Decker L, Allali G, Beauchet O. Development of a short form of mini-mental state examination for the screening of dementia in older adults with a memory complaint: a case control study. BMC Geriatr. 2011;11:59.

30. Hewitt D. Age-related hearing loss and cognitive decline: you Haven't heard the half of it. Front Aging Neurosci. 2017;9:112.

31. Jayakody DMP, Friedland PL, Nel E, Martins RN, Atlas MD, Sohrabi HR. Impact of Cochlear implantation on cognitive functions of older adults: pilot test results. Otol Neurotol. 2017;38(8):e289-95.

32. Jayakody DMP, Friedland PL, Eikelboom RH, Martins RN, Sohrabi HR. A novel study on association between untreated hearing loss and cognitive functions of older adults: baseline non-verbal cognitive assessment results. Clin Otolaryngol. 2018;43(1):182-91.

33. Jette AM. The Impact of Hearing Loss on Physical Functioning. In: Hearing Loss and Healthy Aging: Workshop Summary. Institute of Medicine and National Research Council. Washington, DC: The National Academies Press; 2014. https://doi.org/10.17226/18735.

34. Kuthubutheen J, Mittmann N, Amoodi H, Qian W, Chen JM. The effect of different utility measures on the cost-effectiveness of bilateral cochlear implantation. Laryngoscope. 2015;125(2):442-7.

35. Lachowska M, Pastuszka A, Glinka P, Niemczyk K. Benefits of cochlear implantation in deafened adults. Audiol Neurootol. 2014;19(Suppl 1):40-4.

36. Ladduwahetty S, Dowell RC, Winton E. Dementia and cochlear implant outcomes: can we screen for dementia effectively using the mini mental state examination in implant candidates? Aust NZ J Au. 2013;33(1):88-102.

37. Laureyns M. Economic impact of hearing loss: the different systems of hearing management. Presentation at IFOS: action for global hearing health. Dubai; 2019. [http://www.ifosuae.com/]. Accessed 24 Jun 2019.

38. Lawrence BJ, Jayakody DMP, Bennett RJ, Eikelboom RH, Gasson N, Friedland PL. Hearing Loss and Depression in Older Adults: A Systematic Review and Meta-analysis. Gerontologist. 2019;60(3):e137-54.

39. Lawton MP. The functional assessment of elderly people. J Am Geriatr Soc. 1971;19(6):465-81.

40. le Nobel GJ, Hwang E, Wu A, Cushing S, Lin VY. Vestibular function following unilateral cochlear implantation for profound sensorineural hearing loss. J Otolaryngol Head Neck Surg. 2016;45(1):38.

41. Lenarz T, Muller L, Czerniejewska-Wolska H, Vallés Varela H, Orús Dotú C, Durko $M$, et al. Patient-related benefits for adults with Cochlear implantation: a multicultural longitudinal observational study. Audiol Neurootol. 2017;22(2):61-73.

42. Lin FR, Metter EJ, O'Brien RJ, Resnick SM, Zonderman AB, Ferrucci L. Hearing loss and incident dementia. Arch Neurol. 2011;68(2):214-20.

43. Lin, F. Hearing loss \& aging: a public health perspective. AudiologyOnline, Article 24436. [http://www.audiologyonline.com]. Accessed 18 Jul 2019.
44. Manrique-Huarte R, Calavia D, Huarte Irujo A, Girón L, Manrique-Rodríguez M. Treatment for hearing loss among the elderly: auditory outcomes and impact on quality of life. Audiol Neurootol. 2016;21(Suppl 1):29-35.

45. McRackan TR, Bauschard M, Hatch JL, Franko-Tobin E, Droghini HR, Nguyen SA, Dubno JR. Meta-analysis of quality-of-life improvement after cochlear implantation and associations with speech recognition abilities. Laryngoscope. 2018;128(4):982-90.

46. Michel JP, Sadana R. "healthy aging" concepts and measures. J Am Med Dir Assoc. 2017;18(6):460-4

47. Moberly A, Houston D, Castellanos I. Non-auditory neurocognitive skills contribute to speech recognition in adults with cochlear implants. Laryngoscope. Investig Otolaryngol. 2016;1(6):154-62.

48. Mosnier I, Bebear JP, Marx M, Fraysse B, Granade GL, Mondain M, et al. Improvement of cognitive function after cochlear implantation in elderly patients. JAMA Otolaryngol Head Neck Surg. 2015;141(5):442-50.

49. Mosnier I, Vanier A, Bonnard D, Lina-Granade G, Truy E, Bordure P, et al. Long-term cognitive prognosis of profoundly deaf older adults after hearing rehabilitation using cochlear implants. J Am Geriatr Soc. 2018;66(8):1553-61.

50. Newman C, Jacobson G, Hug G, Weinstein B, Malinoff R. Practical method for quantifying hearing aid benefit in older adults. J Am Acad Audiol. 1991; 2(2):70-5.

51. Noble W, Tyler RS, Dunn CC, Bhullar N. Younger- and older-age adults with unilateral and bilateral cochlear implants: speech and spatial hearing selfratings and performance. Otol Neurotol. 2009;30(7):921-9.

52. Orgeta V, Mukadam N, Sommerlad A, Livingston G. The lancet commission on dementia prevention, intervention, and care: a call for action. Ir J Psychol Med. 2019;36(2):85-8.

53. Panza F, Solfrizzi V, Seripa D, Imbimbo BP, Capozzo R, Quaranta N, et al. Age-related hearing impairment and frailty in Alzheimer's disease: interconnected associations and mechanisms. Front Aging Neurosci. 2015;7: 113.

54. Philip Rajan D, Siti Sabzah MH, Zulkiflee S, Tengku Mohamed I, Kumareysh Vijay $\mathrm{V}$, Iskandar $\mathrm{H}$, et al. Surgical and functional outcomes of cochlear implantation in post-lingual and cross-over patients: first 5-year review of the National Ministry of health Malaysia cochlear implant programme. Med J Malaysia. 2018;73(6):393-6.

55. Pichora-Fuller MK, Mick P, Reed M. Hearing, cognition, and healthy aging: social and public health implications of the links between agerelated declines in hearing and cognition. Sem in Hear. 2015;36(3): 122-39.

56. Podsiadlo D, Richardson S. The timed "up \& go": a test of basic functional mobility for frail elderly persons. J Am Geriatr Soc. 1991;39(2):142-8.

57. Poissant SF, Beaudoin F, Huang J, Brodsky J, Lee DJ. Impact of cochlear implantation on speech understanding, depression, and loneliness in the elderly. J Otolaryngol Head Neck Surg. 2008;37(4):488-94.

58. Population Reference Bureau. 2019 World Population Data Sheet. (2019) https://www.prb.org/population-change/. Accessed 1 Dec 2019.

59. Prince MJ, Wu F, Guo Y, Gutierrez Robledo LM, O'Donnell M, Sullivan R, Yusuf S. The burden of disease in older people and implications for health policy and practice. Lancet. 2015;385(9967):549-62.

60. Psarros C, Cantle Moore, R. Shaping Support for Elderly Cochlear Implant Recipients - The Role of Data Logging and LENA Presentation. Toronto, Canada, The 14th International Conference on Cochlear Implants 2016 May 14. [https://www.acialliance.org/page/DataLoggingConf]. Accessed 10 Jul 2019.

61. Ramakers GG, Smulders YE, van Zon A, Kraaijenga VJ, Stegeman I, Van Zanten GA, Grolman W. Agreement between health utility instruments in cochlear implantation. Clin Otolaryngol. 2016;41(6):737-43.

62. Safdar N. Non-Auditory Verbal and Cognitive Skills in Cochlear Implant Users: Effects of Hearing Loss and Relations to Outcomes. PhD thesis. 2017. [https://kb.osu.edu/bitstream/handle/1811/80514/SafdarNatalie_ undergradthesis.pdf? sequence=4\&isAllowed=y]. Accessed 4 Jul 2019.

63. Sarant JZ, Harris DC, Busby PA, Maruff P, Schembri AJ, Dowell RC, Briggs R. The effect of cochlear implants on cognitive function in older adults: Initial baseline and 18-month follow up results for a prospective international longitudinal study. Front Neurosci. 2019;13:789.

64. Schaefer S, Henderson L, Graham J, Broomfield S, Cullington H, Schramm D, Waltzman S, Bruce I. Review of outcomes and measurement instruments in cochlear implantation studies. Cl Int. 2017;18(5):237-9.

65. Sheikh JI, Yesavage JA. Geriatric depression scale (GDS): recent evidence and development of a shorter version. Clin Gerontol. 1986;5:165-73. 
66. Sonnet MH, Montaut-Verient B, Niemier JY, Hoen M, Ribeyre L, PariettiWinkler C. Cognitive abilities and quality of life after Cochlear implantation in the elderly. Otol Neurotol. 2017 Sep;38(8):e296-301.

67. Souza VC, Lemos SM. Tools for evaluation of restriction on auditory participation: systematic review of the literature [review]. Codas. 2015; 27(4):400-6.

68. Taljaard DS, Olaithe M, Brennan-Jones CG, Eikelboom RH, Bucks RS. The relationship between hearing impairment and cognitive function: a metaanalysis in adults. Clin Otolaryngol. 2016;41(6):718-29.

69. Thomson RS, Auduong P, Miller AT, Gurgel RK. Hearing loss as a risk factor for dementia: a systematic review. LIO. 2017;2(2):69-79.

70. Tombaugh T. Trail making test a \& B: normative data stratified by age and education. Arch Clin Neuropsychol. 2004;19:203-14.

71. UKCISG. Criteria of candidacy for unilateral Cochlear implantation in Postlingually deafened adults I: theory and measures of effectiveness. Ear Hear. 2004;25:310-35.

72. Välimaa T. Speech perception and auditory performance in hearingimpaired adults with a multichannel Cochlear implant. PhD Dissertation. University of Oulu, Finnland Dept ORL; 2002.

73. Völter C, Götze L, Dazert S, Falkenstein M, Thomas JP. Can cochlear implantation improve neurocognition in the aging population? Clin Interv Aging. 2018;13:701-12.

74. World Health Organization. Action for Hearing Loss: Brochure. (2017) [https:/www.who.int/pbd/deafness/world-hearing-day/WHD2017Brochure. pdf?ua=1] Accessed 24 Jun 2019.

75. Yang Z, Cosetti M. Safety and outcomes of cochlear implantation in the elderly: a review of recent literature. J Otol. 2016;11(1):1-6.

76. Zhang J, Tyler R, Ji H, Dunn C, Wang N, Hansen M, Gantz B. Speech, spatial and qualities of hearing scale (SSQ) and spatial hearing questionnaire (SHQ) changes over time in adults with simultaneous Cochlear implants. Am J Audiol. 2015;24(3):384-97.

\section{Publisher's Note}

Springer Nature remains neutral with regard to jurisdictional claims in published maps and institutional affiliations.

Ready to submit your research? Choose BMC and benefit from:

- fast, convenient online submission

- thorough peer review by experienced researchers in your field

- rapid publication on acceptance

- support for research data, including large and complex data types

- gold Open Access which fosters wider collaboration and increased citations

- maximum visibility for your research: over $100 \mathrm{M}$ website views per year

At $\mathrm{BMC}$, research is always in progress.

Learn more biomedcentral.com/submissions 